\title{
LEY GENERAL DE LOS DEREGHOS DE NIÑAS, NIÑOS Y ADOLESGENTES 2014 Y SU REGLAMENTO 2015
}

\author{
Nuria GONZÁLEZ MARTíN* \\ María Elena MiRANDA DELGADO** \\ Daniela RAMÍREZ RAYO***
}

SUMARIO: Nota aclaratoria. I. Estructura y contenido de la Ley General de los

Derechos de Niñas, Niños y Adolescentes. II. Aciertos y desaciertos. III. Estructura y contenido del Reglamento de la Ley General de los Derechos de Niñas, Niños y Adolescentes. IV. Aciertos y desaciertos. V. Conclusiones.

\section{NOTA ACLARATORIA}

El presente comentario legislativo asume la responsabilidad de hacerse desde la perspectiva de un análisis formal, en donde se inicia con una descripción del contenido y finaliza con una serie de comentarios en torno a algunos aciertos y desaciertos - que vislumbran las autoras - y que cubre tanto la Ley General de los Derechos de Niñas, Niños y Adolescentes (LGDNNA) como su reciente Reglamento (RLGDNNA).

Por lo planteado, damos cuenta al lector que no se abordarán en esta oportunidad los antecedentes de la construcción de la categoría de sujeto de las niñas, niños y adolescentes, y tampoco se hará referencia a la problemática cultural que dicha categorización ha supuesto en términos de la armonización de la Convención de Naciones Unidas de 1989 sobre los Derechos del Niño con la legislación nacional o estatal mexicana. Para finalizar, alentamos a aquellos lectores que quieran ampliar el conocimien-

* Doctora en derecho por la Universidad Pablo de Olavide de Sevilla, España. Investigadora titular en el Instituto de Investigaciones Jurídicas de la UNAM, PRIDE D, SNI nivel III; Visiting Fellow en Stanford Law School 2012-2016.

** Maestra en derecho internacional por Cornell University y maestra en ciencias jurídicas por la Universidad Abat Oliva CEU de Barcelona. Actualmente doctorando en la Universidad Panamericana Guadalajara y profesora en derecho internacional.

**** Maestra en derecho por la Facultad de Derecho de la Universidad Nacional Autónoma de México.

Boletín Mexicano de Derecho Comparado nueva serie, año XLIX, núm. 146, mayo-agosto de 2016, pp. 345-374.

D. R. C 2016. UNAM, Instituto de Investigaciones Jurídicas. 
Esta revista forma parte del acervo de la Biblioteca Jurídica Virtual del Instituto de Investigaciones Jurídicas de la UNAM

to de dicha normativa a que se acerquen a los antecedentes legislativos que dicha ley y su reglamento han tenido para poder visualizar un panorama más amplio sobre la derivación de la normativa aprobada.

\section{ESTRUCTURA Y GONTENIDO DE LA LEY GENERAL DE LOS DereGHOS DE NiÑAS, NiÑos y AdOleSGENTES}

El 4 de diciembre de 2015 fue publicado en el Diario Oficial de la Federación $(D O F)$ el decreto por el que se expide la Ley General de los Derechos de Niñas, Niños y Adolescentes y se reforman diversas disposiciones de la Ley General de Prestación de Servicios para la Atención, Cuidado y Desarrollo Integral Infantil.

Como acabamos de expresar, en este apartado el presente comentario legislativo busca, en primer término, exponer la estructura y contenido general de la LGDNNA, para pasar después a mencionar, en otro apartado, lo que consideramos son aciertos y desaciertos de la normativa.

Es preciso comentar que con la publicación de esta nueva ley general, y conforme a lo previsto en el artículo cuarto transitorio del propio decreto, queda abrogada la anterior Ley para la Protección de los Derechos de Niñas, Niños y Adolescentes, vigente desde su publicación en 2000, y que tenía una extensión mucho menor, de sólo 56 artículos, en comparación con la recientemente publicada, que se compone de 154 artículos divididos en seis títulos, conforme al siguiente orden:

- Título primero, "Disposiciones generales", cuyo contenido se encuentra en los primeros doce artículos de la ley;

- Título segundo, "De los derechos de niñas, niños y adolescentes", que es el más extenso de los títulos, y de ahí que se encuentra dividido en diecinueve capítulos, que abarcan del artículo 13 al 101.

- Título tercero, "De las obligaciones", cuyo contenido se contempla del artículo 102 al 106.

- Título cuarto, denominado "De la protección de niñas, niños y adolescentes", con un capítulo único dedicado a regular los centros de asistencia social en los artículos 107 al 113.

- Título quinto, denominado "De la protección y restitución integral de los derechos de niñas, niños y adolescentes", segundo en extensión, con seis capítulos, que van del artículo 114 al 145. 
Esta revista forma parte del acervo de la Biblioteca Jurídica Virtual del Instituto de Investigaciones Jurídicas de la UNAM

- Título sexto, que contempla las infracciones administrativas dentro de un capítulo único, que va de los artículos 146 al 154.

Desde su primer artículo, la ley establece el carácter de orden público, interés social y observancia general en todo el territorio nacional; asimismo, define como su objeto primordial el reconocimiento de niñas, niños y adolescentes como titulares directos de derechos humanos; esto último en concordancia con la Constitución y los tratados internacionales en la materia.

Establece la responsabilidad del Estado mexicano no sólo como garante del pleno ejercicio de los derechos de menores, sino como agente promotor de su respeto y protección si fueron vulnerados, para lo que prevé la creación del Sistema Nacional de Protección Integral de los Derechos de Niñas, Niños y Adolescentes. Siendo que las autoridades federales, de las entidades federativas, municipales y de las demarcaciones territoriales del Distrito Federal, en el ámbito de sus respectivas competencias, están obligadas a coordinarse y a adoptar las medidas necesarias para garantizar estos derechos a todas las niñas, niños y adolescentes sin discriminación de ningún tipo o condición.

Son principios rectores y orientadores de la política nacional en materia de derechos de niñas, niños y adolescentes, entre otros: el derecho a la vida, a la supervivencia, al desarrollo, y el acceso a una vida libre de violencia; la universalidad, interdependencia, indivisibilidad, progresividad e integralidad de los derechos de la niñez; el interés superior de la niñez; la no discriminación y la inclusión de los niños; la interculturalidad y la corresponsabilidad de los miembros de la familia, la sociedad y las autoridades.

En el artículo quinto se determinan los parámetros de edad, estableciendo que se tratará de niñas y niños cuando son menores de doce años, y serán adolescentes una vez cumplidos los doce hasta los dieciocho. Ahí mismo se establece una presunción de minoridad en beneficio; es decir, ante la duda de si se trata de una persona mayor de dieciocho años de edad, se presumirá que es adolescente, y en caso de duda de si se trata de una persona mayor o menor de doce años, se presumirá que es niña o niño, gozando así de un grado mayor de protección de la ley.

Destacan también otras definiciones legales, urgidas y necesarias, tales como acciones afirmativas; acogimiento residencial; discriminación 
Esta revista forma parte del acervo de la Biblioteca Jurídica Virtual del Instituto de Investigaciones Jurídicas de la UNAM

múltiple; familia de acogida y la familia de acogimiento preadoptivo; igualdad sustantiva y la protección integral, así como los conceptos de representación coadyuvante, representación originaria y representación en suplencia.

El título segundo, "De los derechos de niñas, niños y adolescentes", marca el objeto primordial de la ley y concentra el grueso del articulado. El listado inicial que se encuentra en el artículo 13 nos permite señalar los artículos concretos que detallan cada uno de los derechos reconocidos a los menores, entendiendo que esta lista se deberá interpretar de manera enunciativa, pero no limitativa:

I. Derecho a la vida, a la supervivencia y al desarrollo; (capítulo primero, artículos del 14 al 16).

II. Derecho de prioridad (capítulo segundo, artículos 17 y 18).

III. Derecho a la identidad (capítulo tercero, artículos del 19 al 21).

IV. Derecho a vivir en familia (capítulo cuarto, artículos del 22 al 35).

V. Derecho a la igualdad sustantiva (capítulo quinto, artículos del 36 al 38).

VI. Derecho a no ser discriminado (capítulo sexto, artículos del 39 al 42).

VII. Derecho a vivir en condiciones de bienestar y a un sano desarrollo integral (capítulo séptimo, artículos del 43 al 45).

VIII. Derecho a una vida libre de violencia y a la integridad personal (capítulo octavo, artículos del 46 al 49).

IX. Derecho a la protección de la salud y a la seguridad social (capítulo noveno, artículos del 50 al 52).

$\mathrm{X}$. Derecho a la inclusión de niñas, niños y adolescentes con discapacidad (capítulo décimo, artículos del 54 al 56)

XI. Derecho a la educación (capítulo 11, artículos del 57 al 59; tres artículos que son largos en extensión con varias fracciones en numeral romano).

XII. Derecho al descanso y al esparcimiento (capítulo 12, artículos 60 y 61).

XIII. Derecho a la libertad de convicciones éticas, pensamiento, conciencia, religión y cultura (capítulo 13, artículos 62 y 63).

XIV. Derecho a la libertad de expresión y de acceso a la información (capítulo 14, artículos del 64 al 70). 
Esta revista forma parte del acervo de la Biblioteca Jurídica Virtual del Instituto de Investigaciones Jurídicas de la UNAM

XV. Derecho de participación (capítulo 15, artículos del 71 al 74).

XVI. Derecho de asociación y reunión (capítulo 16, artículo único 75).

XVII. Derecho a la intimidad (capítulo 17, artículos del 76 al 81).

XVIII. Derecho a la seguridad jurídica y al debido proceso (capítulo 18, artículos del 82 al 88).

XIX. Derechos de niñas, niños y adolescentes migrantes (capítulo 19, artículos del 89 al 101).

XX. Derecho de acceso a las tecnologías de la información y comunicación, así como a los servicios de radiodifusión y telecomunicaciones, incluido el de banda ancha e Internet, en términos de lo previsto en la Ley Federal de Telecomunicaciones y Radiodifusión. Para tales efectos, el Estado establecerá condiciones de competencia efectiva en la prestación de dichos servicios.

El capítulo décimo noveno dedica trece artículos a la situación jurídica de niñas, niños y adolescentes migrantes, lo que realmente constituye una novedad, necesaria y esperada. Del artículo 89 hasta el 101 se prevén medidas especiales de protección que las autoridades de todos los órdenes de gobierno deberán adoptar para garantizar los derechos de los menores migrantes, lo que harán con independencia de su nacionalidad o de una situación migratoria irregular, y así, expresamente, se establece que en ningún caso preconfigurará por sí misma la comisión de un delito, en concordancia con la Ley de Migración.

La ley establece claramente que durante el procedimiento administrativo migratorio al que estén sujetos niñas, niños y adolescentes migrantes, las autoridades deberán respetar las garantías de debido proceso y desempeñarán sus funciones teniendo como consideración primordial el principio del interés superior de la niñez, lo que implica tomar en cuenta sus opiniones, privilegiando la reunificación familiar, excepto cuando ello sea contrario a su interés superior o voluntad.

Entre las autoridades involucradas en estos procesos se encuentran el Instituto Nacional de Migración, encargado de determinar la condición migratoria del menor, de conformidad con la Ley de Migración y su Reglamento; el Sistema Nacional DIF o sistema de las entidades, que deberán adoptar las medidas correspondientes para brindar la protección debida, entre ellas la habilitación de espacios de alojamiento o albergues para recibir a los menores migrantes donde se les brinde 
Esta revista forma parte del acervo de la Biblioteca Jurídica Virtual del Instituto de Investigaciones Jurídicas de la UNAM

la atención adecuada. Corresponde a la Secretaría de Relaciones Exteriores coordinarse con el Instituto Nacional de Migración y con los Sistemas DIF para garantizar la asistencia social y protección consular de mexicanos menores migrantes que se encuentran en el extranjero en proceso de repatriación.

Se impone al Sistema Nacional DIF el diseño y administración de una base de datos de niñas, niños y adolescentes migrantes extranjeros no acompañados, y detallar las causas de su migración, vínculos familiares y factores de riesgo, que deberá ser compartida con la Procuraduría Federal de Protección de Niñas, Niños y Adolescentes. Finalmente, se establece que las autoridades podrán hacer el reconocimiento de condición de refugiado o de asilo, observando siempre las garantías de seguridad y privacidad, a fin de adoptar medidas de protección especial. Y en todo caso queda prohibido devolver, expulsar, deportar, retornar, rechazar en frontera o no admitir, a un menor migrante cuando su vida, su seguridad y/o su libertad estén en peligro.

En un capítulo único del título tercero se establecen las obligaciones de quienes ejercen la patria potestad, tutela o guarda y custodia de niñas, niños y adolescentes, del que queremos destacar una serie de pautas.

Según el artículo 103, las siguientes obligaciones las tienen tanto personas físicas como instituciones públicas que tengan bajo su guarda o custodia a menores:

I. Garantizar los derechos alimentarios, el libre desarrollo de la personalidad y el ejercicio de los derechos de menores.

II. Su registro oportuno.

III. Asegurar que cursen la educación obligatoria.

IV. Impartir dirección y orientación apropiadas.

V. Asegurar un entorno afectivo, comprensivo y sin violencia.

VI. Fomentar en niñas, niños y adolescentes el respeto a todas las personas $\mathrm{y}$ bienes.

VII. Protegerles contra toda forma de violencia y maltrato.

VIII. Abstenerse de cualquier atentado contra su integridad física, psicológica, o actos que menoscaben su desarrollo integral. Castigo físico.

IX. Considerar la opinión del menor. 
Esta revista forma parte del acervo de la Biblioteca Jurídica Virtual del Instituto de Investigaciones Jurídicas de la UNAM

Cuando los menores han sido privados de cuidado parental o familiar, son canalizados a centros de asistencia social, donde permanecen hasta que su situación legal sea definida. Mientras están bajo el cuidado de estas instituciones, la ley busca garantizar el cumplimiento de sus derechos estableciendo lineamientos generales de operación de estos centros y determinando autoridades competentes para labores de supervisión. Los centros de asistencia social donde se protege a los menores son regulados en el título cuarto en un capítulo único, que consta de siete artículos (107-113).

Es el artículo 112 el que establece que serán las procuradurías de protección de las entidades federativas, en coordinación con la Procuraduría de Protección Federal, las autoridades competentes para autorizar, registrar, certificar y supervisar los centros de asistencia social.

También prevé la conformación de un Registro Nacional de Centros de Asistencia Social del Sistema Nacional DIF, muy necesario para la concentración de información actualizada, misma que deberá ser entregada semestralmente por estos centros a las procuradurías estatales, quienes a su vez deberán informar a la Procuraduría de Protección Federal. La ley establece que este Registro deberá ser público y consultable en la página de internet del Sistema Nacional DIF.

La ley pone especial énfasis en temas de atención integral y multidisciplinaria, de una infraestructura que contemple las medidas de seguridad que garanticen la comodidad, higiene, así como un espacio idóneo de acuerdo con la edad, el sexo o la condición física o mental de los alojados. Otro tema muy presente en la ley es procurar un entorno que provea los apoyos necesarios para fomentar la inclusión de niñas, niños y adolescentes con discapacidad.

Resulta fundamental que los menores cuenten con un expediente completo para efectos de que su situación sea revisada y valorada de manera particular, con miras a facilitar su reincorporación familiar o social, de igual forma que se garantice la protección de sus datos personales.

En el título quinto, en el artículo 127, se establecen la conformación y bases de operación del Sistema Nacional de Protección Integral, integrado por representantes del Poder Ejecutivo federal, entidades federativas, organismos públicos y representantes de la sociedad civil nombrados por el Sistema en los términos del reglamento de la Ley mediante convocatoria pública. 
Esta revista forma parte del acervo de la Biblioteca Jurídica Virtual del Instituto de Investigaciones Jurídicas de la UNAM

Estará presidido por el presidente de la República, y contará con la presencia de los secretarios de Gobernación, Relaciones Exteriores, Hacienda y Crédito Público, Desarrollo Social, Educación Pública, Salud, Trabajo y Previsión Social, y titular del Sistema Nacional DIF.

Las entidades federativas se verán representadas por los gobernadores de los estados, y el jefe de Gobierno del Distrito Federal.

Los organismos públicos cuentan con la presencia del fiscal general de la República, el presidente de la Comisión Nacional de los Derechos Humanos, y el comisionado presidente del Instituto Federal de Telecomunicaciones.

Serán invitados permanentes a las sesiones las mesas directivas de las cámaras de Diputados y Senadores del Congreso de la Unión, un representante del Poder Judicial de la Federación, así como representantes de la Comisión Nacional de Tribunales Superiores de Justicia de los Estados Unidos Mexicanos, las asociaciones de municipios, legalmente constituidas, quienes intervendrán con voz pero sin voto.

Finalmente, en el título sexto, denominado "De las infracciones administrativas", se prevé un capítulo único, donde se establecen las infracciones y sanciones aplicables a servidores públicos federales. Mientras que serán las leyes locales de cada una de las entidades federativas las encargadas de prever tanto infracciones y sanciones como los procedimientos para su imposición e impugnación, así como las autoridades competentes para ello.

Quedarán sujetos a las sanciones administrativas los servidores públicos federales que, en el ejercicio de sus funciones o actividades o con motivo de ellas, indebidamente impidan el ejercicio de algún derecho o nieguen la prestación del servicio al que están obligados; cuando conozcan de la violación de algún derecho a alguna niña, niño o adolescente, e indebidamente se abstengan de hacerlo del conocimiento de la autoridad competente o propicien, toleren o se abstengan de impedir cualquier tipo de abuso, acoso, agresión, daño, intimidación, violencia, maltrato o perjuicio de que tengan conocimiento, en contra de niñas, niños y adolescentes.

\section{ACIERTOS Y DESACIERTOS}

En primer lugar, debemos considerar que siempre será un acierto que la legislación doméstica refleje los acuerdos internacionales asumidos en tratados de los que México es parte. En este sentido, y acorde a la magna 
Esta revista forma parte del acervo de la Biblioteca Jurídica Virtual del Instituto de Investigaciones Jurídicas de la UNAM

reforma constitucional en materia de derechos humanos de junio de 2011, la nueva ley define como su objeto primordial el reconocimiento de niñas, niños y adolescentes como titulares directos de derechos humanos, lo que hace posible que el Estado asuma su representación en aras de protegerlos. Consideramos de suma importancia que la ley haga hincapié en la interdependencia, indivisibilidad, progresividad e integralidad de los derechos de la niñez. Se debe destacar que la ley establece expresamente que el principio rector del interés superior de la niñez deberá ser considerado de manera primordial en la toma de decisiones en todo asunto relacionado con derechos de los menores, y ello es constantemente reiterado a lo largo de todo el texto legal, claramente en especial sintonía con la Convención sobre los Derechos del Niño de 1989. Confiemos que no sólo sea considerado en el papel y se implemente a carta cabal en la práctica.

En segundo lugar, y en relación con el carácter de orden público, interés social y observancia general de la ley en todo el territorio nacional, sabemos que las entidades federativas protegen celosamente la reserva constitucional contenida en el artículo 124, gracias a la cual la materia familiar es tradicionalmente local, y, por lo tanto, pudieran sucumbir ante la tentación de que su legislatura emita una ley espejo. Lo importante en todo caso es que desde su entrada en vigor quedan protegidos los derechos de los menores, conforme a los términos de esta ley, en toda la República.

En tercer lugar, la ley introduce con acierto algunos conceptos que determinan el camino a seguir. Tal es el caso de la presunción de minoridad en beneficio cuando hay duda respecto de la edad del menor; establecer sin ambigüedades la igualdad sustantiva entre niños y niñas definida dentro del texto de la ley en el artículo 4o., fracción XIV: "el acceso al mismo trato y oportunidades para el reconocimiento, goce o ejercicio de los derechos humanos y las libertades fundamentales"; y de manera reiterativa se busca fomentar la inclusión de niñas, niños y adolescentes con discapacidad.

Como cuarto acierto, consideramos que esta nueva ley dedique tres títulos (el cuarto, quinto y sexto), a regular a los centros de asistencia social, la restitución integral de los derechos de los niños y adolescentes y las infracciones administrativas, temas no cubiertos de forma directa en la ley anterior.

En quinto lugar, es un importante avance en la materia de protección de los menores la creación del Registro Nacional de Centros de Asistencia 
Esta revista forma parte del acervo de la Biblioteca Jurídica Virtual del Instituto de Investigaciones Jurídicas de la UNAM

Social del Sistema Nacional DIF y entregas semestrales por los centros de asistencia social a las procuradurías estatales, quienes a su vez deberán informar a la Procuraduría de Protección Federal. Serán las procuradurías de protección de las entidades federativas, en coordinación con la Procuraduría de Protección Federal, las autoridades competentes para autorizar, registrar, certificar y supervisar los centros de asistencia social.

En sexto lugar, resulta atinada la atención directa en el capítulo décimo noveno al delicado tema de la migración de menores dentro de nuestro territorio nacional. En lo relativo a los derechos de los menores migrantes, la ley atiende los dos polos del tema:

a. Por un lado, protege la atención y cuidado que debe brindarse a los menores extranjeros que de suyo se encuentran en una situación más que vulnerable, imponiendo al Sistema Nacional DIF el diseño y administración de una base de datos de niñas, niños y adolescentes migrantes extranjeros no acompañados, en el que se detallen las causas de su migración, vínculos familiares y factores de riesgo. Esta plataforma informativa deberá ser compartida con la Procuraduría Federal de Protección de Niñas, Niños y Adolescentes.

b. Por otro lado, busca una coordinación efectiva entre la Secretaría de Relaciones Exteriores, el Instituto Nacional de Migración y los Sistemas DIF para garantizar la asistencia social y protección consular de mexicanos menores migrantes que se encuentran en el extranjero en proceso de repatriación.

Entrando en el contexto de los posibles desaciertos, tenemos en primer lugar, una falta de orden legislativo, que se encuentra en relación con el concepto de "protección integral", al no formar parte de la lista de principios rectores de la ley del artículo 6o., pero mencionado en el artículo 2o., y definido en el 4o., fracción XX, se la siguiente manera:

Protección Integral: Conjunto de mecanismos que se ejecuten en los tres órdenes de gobierno con el fin de garantizar de manera universal y especializada en cada una de las materias relacionadas con los derechos humanos de niñas, niños y adolescentes de conformidad con los principios rectores de esta Ley, la Constitución Política de los Estados Unidos Mexicanos y los tratados internacionales de los que el Estado mexicano forma parte. 
Esta revista forma parte del acervo de la Biblioteca Jurídica Virtual del Instituto de Investigaciones Jurídicas de la UNAM

En segundo lugar, y en relación con la regulación de los centros de atención de menores, establece bases generales de operación para casas hogar, albergues u orfanatos, sean del sector público o privado. En este sentido, la ley les impone estándares muy altos, los cuales sólo se podrán cumplir si las partidas presupuestarias que se les otorguen son suficientes para cubrir la obligación legal "de ofrecer una atención integral y multidisciplinaria" y para que puedan efectivamente contar con una infraestructura que contemple las "medidas de seguridad que garanticen la comodidad, higiene, así como un espacio idóneo para los menores alojados de acuerdo a su edad, sexo o condición física o mental".

En tercer lugar, y ante el rubro dedicado al Sistema Nacional de Protección Integral de los Derechos de Niñas, Niños y Adolescentes, creado por la ley como una importante plataforma para generar políticas públicas nacionales, pudiera encontrar en su misma conformación trabas suficientes para operar de manera eficiente, estamos pensando en el inconveniente que pudiera darse al estar integrado por altos funcionarios del Ejecutivo federal, por los gobernadores de los estados y otros representantes de los poderes federales; dado que "deberá reunirse cuando menos dos veces al año y que para sesionar válidamente requerirá un quórum de la mayoría de sus miembros y la asistencia del Presidente de la República”. Pensamos que pudiera convertirse en un órgano ineficaz quedando nuevamente el interés de la minoridad en un segundo plano.

\section{ESTRUCTURA Y CONTENIDO DEL REGLAMENTO DE LA LEY GENERAL DE LOS DEREGHOS DE NiÑAS, NiÑOS Y ADOLESGENTES}

Una vez relatada la estructura y aquellos pros y contras de la Ley General de los Derechos de Niñas, Niños y Adolescentes, es el turno de comentar su Reglamento, RLGDNNA, el cual fue publicado en el DOF el 2 de diciembre de 2015 .

Cabe mencionar que la anterior Ley para la Protección de los Derechos de las Niñas, Niños y Adolescentes no contaba con un reglamento que ampliara sus disposiciones.

La primera cuestión que llama la atención de la nueva LGDNNA es que su artículo quinto transitorio señala que las disposiciones reglamentarias deberán ser expedidas a los ciento ochenta días naturales siguientes 
Esta revista forma parte del acervo de la Biblioteca Jurídica Virtual del Instituto de Investigaciones Jurídicas de la UNAM

a la entrada en vigor de la propia Ley, por lo que el Reglamento, objeto de este comentario, debió ser expedido a los seis meses posteriores al 5 de diciembre de 2014, fecha en la que entró en vigor la LGDNNA, y no a un año de su publicación. El Reglamento, como cualquier disposición de su naturaleza, tiene como función primordial, facilitar la aplicación de la Ley de la cual deriva, por lo que es preocupante la tardanza en su publicación.

Con relación a su estructura, el RLGDNNA consta de 114 artículos divididos en diez títulos, además de ocho artículos transitorios. Los títulos en los que se divide el Reglamento son los siguientes:

- Título primero "De las disposiciones generales". Consta de sólo cuatro artículos, en los cuales se señala el objeto del Reglamento, algunos conceptos complementarios a los que define la Ley y señala las dependencias encargadas de la interpretación del propio Reglamento.

- Título segundo, relativo al "Sistema nacional de protección integral", que se divide en tres capítulos relativos a la integración y funcionamiento del propio sistema, y que abarca los artículos del 6 al 23.

- Título tercero "Del Programa Nacional y Evaluación de las Políticas Vinculadas con la Protección de los Derechos de Niñas, Niños y Adolescentes", dividido únicamente en dos capítulos, referentes a disposiciones generales del Programa y a la evaluación de las políticas vinculadas con la protección de los derechos de niñas, niños y adolescentes; artículos del 24 al 33.

- Título cuarto "Del Sistema Nacional de Información, Registros Nacionales y Bases de Datos de Niñas, Niños y Adolescentes", que se divide en cinco capítulos referentes a diversos registros y bases de datos, que permitirán un mejor conocimiento de las situaciones actuales de niñas, niños y adolescentes en diversas condiciones, como son los niños que se encuentran en centros de asistencia social, de niños migrantes o niños en procesos de adopción, entre otros; consta de los artículos del 34 al 45.

- Título quinto, relativo a la "Protección y restitución de derechos de niñas, niños y adolescentes", que contiene un capítulo único, referente a las acciones de protección de niñas, niños y adolescentes; que a su vez consta de únicamente tres artículos (45 al 47). 
Esta revista forma parte del acervo de la Biblioteca Jurídica Virtual del Instituto de Investigaciones Jurídicas de la UNAM

- Título sexto "De las medidas de protección", dividido en cinco capítulos, que abarcan los artículos 48 al 70, referidos a las medidas de protección especial, a las medidas urgentes, al acogimiento residencial, de los centros de asistencia social y de las familias de acogida.

- Título séptimo, referido a los "Procedimientos de adopción", que contienen tan sólo tres capítulos, que se refieren a la emisión de los certificados de idoneidad, del acogimiento preadoptivo y de la autorización de profesionales en materia de trabajo social y psicología o carreras afines para intervenir en los procedimientos de adopción; consta de los artículos del 71 al 85.

- Título octavo, que reglamenta la adopción internacional, a través de sus dos capítulos, referentes a la adopción de niños residentes en México y de niños residentes en el extranjero (artículos 86 al 104).

- Título noveno, "De las niñas, niños y adolescentes migrantes", que en pocas palabras señala diversas atribuciones al Instituto Nacional de Migración, relativas a los procedimientos administrativos migratorios en los que se involucren niñas y niños y adolescentes; consta de sólo siete artículos, del artículo 105 al 111.

- Título décimo, "De las sanciones administrativas", que consta de los últimos tres artículos, donde se señalan las autoridades facultadas para la aplicación de las sanciones establecidas en la propia ley.

El primer artículo del Reglamento establece que éste es de orden público, de interés social y de observancia general, y que tiene por objeto regular las atribuciones de la administración pública federal a efecto de respetar, promover, proteger y garantizar los derechos de niñas, niños y adolescentes.

Posteriormente, en el artículo segundo se señalan conceptos utilizados en el reglamento, entre los que destaca la creación de la Secretaría Ejecutiva como un órgano administrativo desconcentrado de la Secretaría de Gobernación, que se encarga de la coordinación operativa del Sistema Nacional de Protección Integral (SNPI).

Cabe recordar que el Sistema Nacional de Protección Integral se integrará con diversos funcionarios del Ejecutivo federal, así como funcionarios de las administraciones locales y de otros representantes de los po- 
Esta revista forma parte del acervo de la Biblioteca Jurídica Virtual del Instituto de Investigaciones Jurídicas de la UNAM

deres federales. Por lo anterior, la Secretaría Ejecutiva funcionará como un órgano de coordinación que garantice la concurrencia de competencias entre los diversos órganos integrantes del Sistema. En otras palabras, podríamos decir que la Secretaría Ejecutiva es un órgano administrativo que organiza las actividades del propio Sistema Nacional de Protección Integral a fin de que sus integrantes lleven a cabo sus actividades de forma coordinada.

Para la organización y funcionamiento del Sistema, la Secretaría Ejecutiva deberá elaborar un manual de organización y operación, que entre otros aspectos debe contener: a) los mecanismos para convocar las sesiones ordinarias y extraordinarias; $b$ ) el contenido de las actas de sesiones, y c) la forma en que se realizarán las invitaciones permanentes a las sesiones, así como la forma de seleccionar a las niñas, niños y adolescentes que participarán en las sesiones.

En lo referente al Manual de Organización y Operación, el artículo quinto transitorio del propio reglamento señala que los lineamientos, acuerdos, protocolos, metodologías y demás disposiciones administrativas de carácter general se deberán emitir en un plazo de ciento ochenta días naturales a partir de la entrada en vigor del Reglamento, por lo que dicho manual, a la fecha de esta publicación no ha sido emitido.

Es importante señalar cómo el Reglamento delega en el Manual de Organización y Operación del Sistema la forma de seleccionar a las niñas, niños y adolescentes que deberán participar en las sesiones, de conformidad con el artículo 127 de la Ley, un tema de gran importancia, al considerarse el mayor ejemplo de cómo en la actualidad las niñas, niños y adolescentes son reconocidos como titulares directos de derechos humanos, y como tales deben ser escuchados en los asuntos que les conciernen. Esperemos que dicho Manual señale un procedimiento efectivo y representativo para la selección de las niñas, niños y adolescentes que deban participar en las sesiones del Sistema; es decir, que el Manual garantice la participación de los menores y que las niñas, niños y adolescentes seleccionados representen la problemática actual de la niñez mexicana.

Asimismo, según el artículo 13, el Sistema deberá integrarse con ocho representantes de la sociedad civil, que serán elegidos mediante convocatoria publicada en el $D O F$, y durarán en su cargo cuatro años. Entre los requisitos que deberán cubrir los aspirantes encontramos: 
Esta revista forma parte del acervo de la Biblioteca Jurídica Virtual del Instituto de Investigaciones Jurídicas de la UNAM

1. Tener residencia permanente en México;

2. No haber sido condenado por la comisión de un delito doloso en el que sujeto pasivo o víctima del mismo haya sido una niña, niño o adolescente;

3. Experiencia mínima de cinco años comprobada en la defensa, promoción de los derechos de la infancia o derechos humanos, y

4. No haber ocupado cargo público ni haber desempeñado cargo de dirección nacional o estatal en algún partido político, por lo menos dos años antes de su postulación.

Es de mencionar que conforme al artículo segundo transitorio, en la primera elección de los representantes de la sociedad civil, cuatro de los ocho representantes serán determinados mediante sistema de insaculación, y durarán en su encargo únicamente dos años.

Por otro lado, el Sistema contará con un consejo consultivo, integrado por veinte integrantes de los sectores público, privado, académico y social, cuyas actividades se encaminarán a la emisión de recomendaciones para la mejor implementación de las políticas públicas, programas y acciones que realice el Sistema Nacional de Protección Integral. Como su propio nombre lo indica, este consejo únicamente podrá aportar opiniones o recomendaciones, pero no tendrá facultad de decisión.

Ahora bien, tanto el Sistema Nacional de Protección Integral como los sistemas locales basarán su actuar en el Programa Nacional, el cual tendrá como base un diagnóstico sobre la situación de los derechos de niñas, niños y adolescentes, y que, en pocas palabras, marcará las líneas de acción en cuanto a la protección y restitución de los derechos de las niñas, niños y adolescentes.

El Programa Nacional será elaborado por la Secretaría Ejecutiva, mediante un proceso de recopilación de información, propuestas y opiniones de los integrantes del Sistema, de las organizaciones de la sociedad civil, de los organismos internacionales, de las niñas, niños y adolescentes, así como, en su caso, de los demás participantes de los sectores público, social, académico y privado (artículo 25).

El anteproyecto del Programa, como lo señala el artículo 27, entre otros aspectos deberá contener lo siguiente: 
Esta revista forma parte del acervo de la Biblioteca Jurídica Virtual del Instituto de Investigaciones Jurídicas de la UNAM

- Las políticas, objetivos, estrategias, líneas de acción prioritarias, metas, indicadores para el ejercicio, respeto, promoción y protección integral de los derechos de las niñas, niños y adolescentes.

- La estimación de recursos, fuentes de financiamiento e instrumentos financieros para la ejecución del Programa.

- Mecanismos que aseguren una ejecución coordinada del Programa Nacional.

- Mecanismos de participación de niñas, niños y adolescentes, y de los sectores público, privado y de la sociedad civil.

- Mecanismos de transparencia y rendición de cuentas.

- Mecanismos de evaluación.

Por otra parte, el título IV señala que entre las atribuciones de la Secretaría Ejecutiva se encuentra la realización y actualización de un Sistema Nacional de Información, que se integra con información estadística relativa a las situación sociodemográfica, la situación de vulnerabilidad y la discapacidad de los derechos de niñas, niños y adolescentes, así como de información que permita evaluar y monitorear la implementación y cumplimiento de los mecanismos establecidos en la Ley y en los tratados internacionales.

Así pues, el Sistema Nacional de Información se compondrá de diversos registros y bases de datos que permitan conocer la situación actual de las niñas, niños y adolescentes que se encuentran en diversas situaciones, tales como los menores sujetos a un procedimiento de adopción, ya sea nacional o internacional; los menores que se encuentran con familias de acogida; los menores que se hallen en centros de asistencia social y menores migrantes, entre otros.

Dentro de estos registros destaca el registro de niñas, niños y adolescentes susceptibles de adopción, que se compone de cuatro bases de datos de suma importancia: 1) de menores susceptibles de ser adoptados; 2) de personas interesadas en adoptar; 3) de los procedimientos de adopción, y 4) de las niñas, niños y adolescentes adoptados (artículo 38).

Cada una de las bases de datos deberá incluir, además de los datos generales de los menores o de las personas interesadas en adoptar, aspectos específicos de su condición, como por ejemplo, en el caso de menores susceptibles de adopción, se debe especificar su condición pedagógica, información social, diagnostico médico y psicológico, además del perfil 
Esta revista forma parte del acervo de la Biblioteca Jurídica Virtual del Instituto de Investigaciones Jurídicas de la UNAM

de atención familiar y requerimiento de atención especial; en cuanto a las personas interesadas en adoptar, se deberá incluir el perfil y el número de las niñas, niños y adolescentes que tienen la capacidad de adoptar, y si cuenta con certificado de idoneidad.

El registro de niñas, niños y adolescentes susceptibles de adopción es de suma importancia, porque se podrá contar con una base de datos que además de determinar el número de menores susceptibles de darse en adopción incluirá datos referentes a la situación física y psicológica del menor, así como la situación escolar, la situación jurídica, el tipo y la severidad de la discapacidad - en su caso - el perfil de necesidades de atención familiar y requerimientos de atención a necesidades, además de que se dará seguimiento, paso a paso, del proceso de adopción, y se tendrá información referente a procesos de adopción no concluidos.

Todos estos tópicos son de gran importancia, en primer lugar, para identificar las necesidades de cada niño, tanto de escolaridad como de atención médica, y luego para poder realizar estadísticas que indiquen qué niños, con qué características, son adoptados en su mayoría, y conocer las causas de adopciones inconclusas, y así, de esta manera, prever acciones que aseguren las mejores condiciones para los menores y garantizar el cumplimiento de sus derechos.

Otra base de datos importante es la referente al seguimiento de los niños ya adoptados, la cual debe contener, entre otros, la fecha de entrega física del menor; en casos de adopción internacional, la fecha en que la niña, niño o adolescente salió del país; el nuevo nombre de la niña, niño o adolescente; el informe de seguimiento postadoptivo y la información de procedimientos previos. Esta base de datos tiene como fin dar un seguimiento de las condiciones del niño adoptado, que permitan conocer su estado de bienestar y el cumplimiento de los principios que enumera la Ley -igualdad sustantiva, participación, interculturalidad, autonomía progresiva, etcétera-.

En este mismo tenor de ideas, y en concordancia con la propia Ley, el capítulo IV del Reglamento impone al Sistema Nacional DIF la administración de bases de datos de niñas, niños y adolescentes migrantes extranjeros, las cuales deben contener los siguientes datos: el nombre completo del menor, su lugar de origen, edad, sexo, media filiación, escolaridad, sitio o zona de cruce fronterizo, número de ocasiones de repatriación, situación de salud, susceptibilidad de recibir protección internacional o 
Esta revista forma parte del acervo de la Biblioteca Jurídica Virtual del Instituto de Investigaciones Jurídicas de la UNAM

complementaria, la identificación de que fue víctima, testigo u ofendido de algún delito en su país de origen, residencia habitual, país de destino o en territorio nacional, las medidas de protección que se le hayan asignado, fecha y lugar en que la autoridad migratoria tuvo contacto con el menor y tipo y severidad de la discapacidad con la que vive.

El registro de menores migrantes permite conocer las situaciones en que se encuentran los menores que tratan de llegar o atravesar nuestro país en búsqueda de mejores condiciones de vida. Como sabemos, la migración es un fenómeno en aumento en nuestro país y en América Latina, debido principalmente a cuestiones de índole económica y social, y este fenómeno alcanza a la niñez dado que cada vez es mayor la cantidad de menores que abandonan su país de residencia y llegan a territorio nacional ya sea con la intención de residir en él o llegar a un tercer país. El Sistema Nacional DIF estima que en 2015 por lo menos 27 mil menores no acompañados cruzaron nuestras fronteras.

Las causas de la migración infantil son muy diversas y realmente preocupantes. Muchos menores abandonan su país de residencia para seguir a sus padres que previamente migraron, o simplemente son menores no acompañados buscando mejores condiciones de vida para ellos y sus familias, encontrando por el camino situaciones de tráfico laboral, sexual, de órganos, entre las muchas y diversas situaciones lamentables en las que se ven inmersos. Los menores migrantes son un sector sumamente vulnerable, y es necesario que el gobierno mexicano implemente acciones de protección de sus derechos; el registro de menores migrantes es un mecanismo que permite realizar estadísticas, y de ahí la implementación de políticas públicas tendentes a la prevención de delitos en contra de menores, y de protección de los derechos humanos; además de que permite identificar situaciones o lugares en donde exista peligro para este sector vulnerable.

El título V del Reglamento señala las atribuciones que tendrá la Procuraduría Federal para hacer cumplir sus obligaciones a quienes ejerzan la patria potestad, guarda y custodia, o cualquier otra persona que tenga bajo su cuidado niñas, niños y adolescentes.

El título VI se refiere propiamente a las medidas que deberán implementar, en el ámbito de sus competencias, la Procuraduría Federal y las procuradurías locales, así como las autoridades federales, estatales y municipales, para la protección de los menores. El título al que nos referimos 
Esta revista forma parte del acervo de la Biblioteca Jurídica Virtual del Instituto de Investigaciones Jurídicas de la UNAM

divide a las medidas de protección en dos grandes grupos: medidas de protección especial y medidas urgentes de protección especial. Las medidas de protección especial son medidas que deben aplicarse en circunstancias o hechos especiales, como algún tipo de discapacidad o menores que son víctimas de violencia, abuso o explotación sexual o laboral, entre otros, y que tienden a restituir o equiparar el cumplimiento de los derechos de las niñas, niños y adolescentes.

Las medidas de protección especial pueden ser todas aquellas acciones que resulten necesarias para salvaguardar los derechos de los menores, y entre ellas se pueden incluir: inclusión en programas de asistencia social, de salud y educativos; órdenes de tratamiento médico, psicológico o psiquiátrico tanto de los menores como de su familia; la separación inmediata de actividades laborales, el acogimiento residencial o la separación inmediata de la persona que ejerza maltrato o violencia.

Para la adopción de medidas de protección, las autoridades deberán argumentar su procedencia y la forma en que preservan los derechos de la niñez, basados en criterios de razonabilidad y progresividad.

Por su parte, las medidas urgentes de protección especial serán implementadas en aquellos casos en que haya un peligro inminente que ponga en riesgo la vida o los derechos del menor, en cuyo caso la Procuraduría Federal ordenará la aplicación de dichas medidas y deberá notificarlo al Ministerio Público, quien a su vez deberá dar aviso al órgano jurisdiccional competente a fin de que éste se pronuncie sobre la cancelación, ratificación o modificación de la medida.

El procedimiento para la adopción de medidas urgentes se encuentra especificado en la Ley; sin embargo, pensamos que dicho procedimiento debió ser ampliado en el propio Reglamento, principalmente en cuanto a la actuación y competencia de la Procuraduría Federal y la intervención de las procuradurías de protección locales.

En lo referente al procedimiento de adopción de medidas urgentes especiales, la Ley señala en su artículo 122, fracciones VI y VII, las atribuciones de la Procuraduría Federal de Protección para solicitar al Ministerio Público la aplicación de dichas medidas, y en casos urgentes decretar por sí misma las medidas urgentes. El Reglamento se limita a señalar que en la solicitud de medidas urgentes, la Procuraduría Federal deberá manifestar los hechos y argumentos que justifiquen la necesidad de dichas medidas, además de establecer que la Procuraduría, en el caso de que ordene 
Esta revista forma parte del acervo de la Biblioteca Jurídica Virtual del Instituto de Investigaciones Jurídicas de la UNAM

por ella misma la aplicación de las medidas, deberá notificar de inmediato al Ministerio Público, y podrá solicitar el auxilio y colaboración de instituciones policiales. El Reglamento no especifica otros elementos formales de la solicitud ni señala en qué casos específicos la Procuraduría podrá dictar por ella misma las medidas de protección.

Cabe mencionar que la Procuraduría Federal no tiene facultad para ejecutar directamente las medidas de protección, sino que su función es coordinar a las dependencias e instituciones que sí tienen facultad para ello. Ante esta situación, pensamos que la atribución de la Procuraduría de dictar por ella misma las medidas de protección urgentes en casos específicos se ve entorpecida, ya que a pesar de que se dicten las medidas con la premura necesaria para evitar o detener la vulneración de derechos, la Procuraduría deberá dar aviso a las instituciones que sí están facultadas para la ejecución de las medidas, lo que puede resultar en un procedimiento lento o ineficaz para la protección de los derechos de niñas, niños y adolescentes.

Posteriormente, en el mismo título VI, el Reglamento desarrolla algunas medidas de protección especial, como el acogimiento residencial y las familias de acogida. El acogimiento residencial, señala la propia ley, es una medida de protección de carácter subsidiario, que consiste en el traslado del menor a un centro de asistencia social a fin de brindarle protección y amparo. Los centros de asistencia social pueden ser de carácter público o privado, y la Procuraduría Federal se encargará de su supervisión a través de visitas que se llevarán a cabo conforme a lo dispuesto en la Ley Federal de Procedimiento Administrativo.

En el capítulo V del título VI se señalan los procedimientos y métodos de regulación de las familias de acogida, una figura jurídica aclamada por años en México, pero sin un reconocimiento y regulación. Las familias de acogida, como su nombre lo indica, son grupos familiares que reciben de forma temporal a niñas, niños o adolescentes a fin de brindarles cuidado, protección, crianza positiva y promoción de bienestar social, hasta que se les asegure una opción permanente con su familia de origen, extensa u adoptiva. Las familias que deseen constituirse como familia de acogida deberán solicitar a la Procuraduría Federal su autorización, mediante una solicitud firmada por quien o quienes serán los responsables de la guarda y custodia de los menores. La Procuraduría, en su caso, deberá expedir la certificación correspondiente. Para obtener 
Esta revista forma parte del acervo de la Biblioteca Jurídica Virtual del Instituto de Investigaciones Jurídicas de la UNAM

dicha certificación como familia de acogida, la familia deberá reunir los mismos requisitos que para obtener el certificado de idoneidad de adopción y, una vez obtenido, deberá acudir a un curso de capacitación, en el cual se les informará sobre los aspectos psicosociales, administrativos y judiciales del acogimiento; además, durante el tiempo del acogimiento deberán rendir informes mensuales y permitir las visitas de verificación realizadas por la propia Procuraduría.

El título VII se refiere a los procedimientos de adopción, y se integra con tres capítulos, referentes a la emisión del certificado de idoneidad, el acogimiento preadoptivo y de la autorización de profesionales en materia de trabajo social y psicología o carreras afines para intervenir en los procedimientos de adopción.

El primer capítulo del título VII, como ya lo indicamos, está dedicado al certificado de idoneidad; es decir, el documento expedido por la Procuraduría de Protección, con la opinión del comité técnico, que señala que las personas que desean adoptar a una niña, niño o adolescente son aptas para tal efecto.

Para la expedición del certificado de idoneidad (artículo 72), y también para obtener la certificación como familia de acogida (artículo 62), se debe cumplir con los siguientes requisitos:

a) Exponer de forma clara y sencilla las razones de su pretensión; b) que la adopción es benéfica para la niña, niño o adolescente que se pretende adoptar; c) tener más de veinticinco años de edad cumplidos al momento que el juez emita la resolución que otorgue la adopción, y tener por lo menos diecisiete años más que el adoptado; d) contar con medios suficientes para proveer la subsistencia y educación de la niña, niño o adolescente que pretenden adoptar; $e$ ) demostrar un modo de vida honesto, así como la capacidad moral y social para procurar una familia adecuada y estable al adoptado; f) no haber sido procesado o encontrarse en un proceso penal por delitos que atenten contra la familia, sexuales o, en su caso, contra la salud, y $g$ ) la demás información que el Sistema Nacional DIF considere necesaria para asegurar y preservar el interés superior de la niñez, mediante acuerdo que publique en el Diario Oficial de la Federación.

Además de lo anterior, las personas solicitantes deberán acudir a un curso de inducción, en el cual se les informará sobre los aspectos psicosociales, administrativos y judiciales de la adopción. 
Esta revista forma parte del acervo de la Biblioteca Jurídica Virtual del Instituto de Investigaciones Jurídicas de la UNAM

El artículo 74 del Reglamento prohíbe la convivencia de las niñas, niños y adolescentes con las personas solicitantes de adopción en tanto éstas no tengan el certificado de idoneidad, a excepción del caso en que el menor y el solicitante sean familiares.

El capítulo segundo se ocupa de una figura novedosa, que es el acogimiento preadoptivo, el cual se refiere al periodo de convivencia entre la niña, niño o adolescente candidato a ser adoptado con la familia solicitante de la adopción, a efecto de confirmar la compatibilidad entre ambos (artículo 77). Para que se dé el acogimiento preadoptivo, los solicitantes ya deben contar con el certificado de idoneidad expedido por la Procuraduría Federal de Protección.

El acogimiento preadoptivo será supervisado por profesionales en materia de psicología y trabajo social, quienes en un periodo de treinta días, contados a partir de que inicie la convivencia, deberán emitir un informe en el cual determinarán si la adopción es favorable para el menor. Si la adopción es favorable, se iniciará el procedimiento de jurisdicción voluntaria de adopción, y si no lo es se iniciará un procedimiento para reincorporar al menor al Sistema Nacional DIF, un tema sumamente delicado pensando en el interés superior del menor. Una vez que cause estado la resolución que determine la adopción, la Procuraduría Federal hará la entrega definitiva de la niña, niño o adolescente y de la documentación del mismo.

El Reglamento es omiso en cuanto al tiempo que deberá durar el acogimiento preadoptivo, además de que no queda totalmente claro si el acogimiento preadoptivo prevalecerá durante el procedimiento de adopción ante el órgano jurisdiccional, o durante el procedimiento de reincorporación al Sistema Nacional DIF.

De acuerdo con el artículo 26 de la Ley, el acogimiento preadoptivo es una fase dentro del proceso de adopción; sin embargo, esta disposición tendría que ser ampliada en el Reglamento, ya que queda en el aire en qué momento del proceso de adopción debe decretarse el acogimiento preadoptivo, por lo que se deberá estar a los procedimientos internos del Sistema Nacional DIF. Según la página electrónica del Sistema Nacional DIF (http://sitios.dif.gob.mx/pdmf/adopciones-2/adopcion-nacional/), posterior a la asignación del menor y la presentación física entre éste y los solicitantes, se iniciará un periodo de convivencia, primero en el lugar donde se encuentra el menor, y posteriormente fuera del centro. El acogimiento 
Esta revista forma parte del acervo de la Biblioteca Jurídica Virtual del Instituto de Investigaciones Jurídicas de la UNAM

preadoptivo dará inicio una vez que los solicitantes asuman todas las obligaciones en cuanto al cuidado y protección del menor.

El título VIII de la adopción internacional se divide en dos capítulos. El primero se refiere a la adopción internacional de niñas, niños y adolescentes con residencia en México, y el segundo capítulo se refiere a la adopción internacional de niñas, niños y adolescentes con residencia en el extranjero.

El artículo 87 del Reglamento señala lo siguiente:

87. La Adopción Internacional en la que México participa como país de origen es aquélla en la que los solicitantes de adopción tienen su residencia habitual en el extranjero y pretenden adoptar una niña, niño o adolescente con residencia en México.

Por su parte, el artículo 100 del mismo Reglamento establece que

100. La Adopción Internacional en la que México participa como país de recepción es aquélla en la que los solicitantes de adopción tienen su residencia habitual en México y pretenden adoptar una niña, niño o adolescente con residencia habitual en el extranjero.

De lo anterior, lo primero que debemos observar es que el concepto de adopción internacional se ve enriquecido al señalar una doble vía para su conformación; así, México, además de ser un país específicamente calificado de emisión de menores, puede ser país de recepción, dependiendo del lugar de procedencia de la niña, niño o adolescente. De esta forma, el concepto de adopción internacional es ampliado para señalar que ésta tiene ese carácter siempre que el menor sujeto a adopción sea desplazado, por ese hecho, del lugar de su residencia habitual, hacia otro Estado. Anteriormente, el concepto de adopción internacional se encontraba reducido, al señalar, como lo hace el Código Civil del Distrito Federal en su artículo 410-E, que la adopción internacional es aquella promovida por extranjeros que tuvieran su residencia habitual fuera del territorio nacional, por lo que se entendía que la adopción internacional únicamente se configuraba cuando México funge como país de origen, omitiendo el caso en que nacionales puedan adoptar un menor extranjero, o a un menor mexicano que se encuentre en un Estado extranjero. 
Esta revista forma parte del acervo de la Biblioteca Jurídica Virtual del Instituto de Investigaciones Jurídicas de la UNAM

Un segundo elemento a resaltar es que estas definiciones tienen como punto de conexión para la configuración de la adopción internacional, no la nacionalidad de las partes, sino la residencia habitual, un tema superado en la comunidad internacional, y que México aún tenía pendiente de afinar.

Para el caso en que México funja como país de origen, corresponde a las autoridades mexicanas velar por la protección de los derechos de la niña, niño o adolescente y garantizar que la adopción no tenga fines ilícitos; para lo anterior, la Ley establece la obligación a cargo del Sistema Nacional DIF de emitir un informe de adoptabilidad; es decir, un documento que contempla los datos de identidad, sociales y familiares del menor, y que debe enviarse a la autoridad central del país de los solicitantes a fin de obtener el acuerdo para la asignación.

De la misma forma, en todos los procedimientos de adopción internacional, los solicitantes, siguiendo los requerimientos establecidos en el Convenio de La Haya del 29 de mayo de 1993 sobre protección de menores en materia de adopción internacional, deberán contar con el certificado de idoneidad, documento expedido por la autoridad central de su lugar de residencia, el cual deberá ser enviado, junto con otros documentos, al Sistema Nacional DIF para su examen. Si posterior al examen del expediente se determina que los solicitantes no cumplen con los requisitos para la adopción internacional, el expediente será devuelto, señalando los requisitos que no son cumplidos y, en su caso, el tiempo para su cumplimiento; si, por el contrario, se determina la procedencia de la adopción internacional, el Sistema Nacional DIF remitirá el expediente al sistema de la entidad que corresponda para que continúe con el trámite.

En el título IX, el Reglamento establece algunas obligaciones y facultades para el Instituto Nacional de Migración. Entre ellos, el artículo 105 ordena al Instituto la creación de protocolos para asegurar que en los procedimientos administrativos migratorios que involucren a niñas, niños y adolescentes se respete el principio del interés superior del menor.

Por último, el título $\mathrm{X}$ del Reglamento está dedicado a las sanciones administrativas. En sus tres únicos artículos, del artículo 112 al 114, señala las dependencias u órganos encargados de la aplicación de las sanciones. En este tenor de ideas, corresponde al Sistema Nacional DIF aplicar las sanciones que establezca la Ley Federal de Procedimiento Administrativo a los profesionales en trabajo social o psicología que intervengan en proce- 
Esta revista forma parte del acervo de la Biblioteca Jurídica Virtual del Instituto de Investigaciones Jurídicas de la UNAM

dimientos de adopción que no cuenten con la autorización, y corresponde a la Secretaría de Gobernación, a través de de las direcciones generales de Radio, Televisión y Cinematografía y de Medios Impresos, aplicar las sanciones a los concesionarios de radio y televisión y de quien dirija medios impresos, por la difusión o transmisión de imágenes, voz, datos o entrevistas que pongan en peligro los derechos de las niñas, niños y adolescentes.

\section{ACIERTOS Y DESACIERTOS}

El Reglamento de la LGDNNA surge de la necesidad de normar la actuación de las entidades de la administración pública federal referente a la protección y preservación de los derechos de las niñas, niños y adolescentes. Para la elaboración del Reglamento, la Secretaría de Gobernación, a través de la Dirección General de Políticas Públicas de Derechos Humanos, solicitó al Fondo de las Naciones Unidas para la Infancia (UNICEF), que realizara algunas propuestas para su inclusión en dicha normativa. En respuesta a dicha solicitud, UNICEF México elaboró una propuesta que incluye, ente otros, los siguientes temas:

- Derecho de participación de niñas, niños y adolescentes y su participación en el SNPI.

- Orientaciones para el desarrollo del Reglamento de la Ley General de Niñas, Niños y Adolescentes (derecho a vivir en familia, adopción y centros de asistencia social).

- Derechos de las niñas, niños y adolescentes migrantes.

- Medidas de protección especial.

Ciertamente, el Reglamento de la LGDNNA toma en cuenta algunas recomendaciones hechas por UNICEF, pero en algunos casos necesita ser ampliado, a fin de desarrollar mejor los conceptos que la Ley ordena.

Por el desarrollo mismo del RLGDNNA, en este apartado no procederemos a enumerar los aciertos y desaciertos de la misma, tal y como hicimos en el apartado relativo a la LGDNNA, sino que comentaremos, en general, cuáles pueden ser sus pros y sus contras.

Con esto en mente, iniciamos por expresar que la Ley, LGDNNA, en numerosas ocasiones, señala la importancia de la participación de las niñas, niños y adolescentes en aquellos asuntos que les afecten; sin embar- 
Esta revista forma parte del acervo de la Biblioteca Jurídica Virtual del Instituto de Investigaciones Jurídicas de la UNAM

go, en este tema el Reglamento se ve un tanto limitado. Según la Ley, las niñas, niños y adolescentes deben tener una participación efectiva en los diversos aspectos relevantes de su desarrollo, como en materia familiar, educativa, cultural, social y comunitaria, entre otros; sin embargo, el Reglamento en pocas ocasiones menciona la participación de los menores y omite señalar procedimientos que permitan su participación.

El Reglamento únicamente establece la participación de las niñas, niños y adolescentes en la integración del Sistema Nacional de Protección Integral y en lo relativo al procedimiento de adopción; no obstante, el Reglamento no amplía la forma en que las niñas, niños y adolescentes podrán tener una participación en estos rubros.

En relación con la participación de las niñas, niños y adolescentes en la integración del Sistema Nacional de Protección, el propio Reglamento delega en el Manual de Organización y Operación la forma de selección de las niñas, niños y adolescentes (artículo 10), pero exceptúa señalar, al menos, los lineamientos que deberá tomar en cuenta dicho Manual, tales como la cantidad de niños que deberán participar, la edad, el origen, la escolaridad, la posición socioeconómica, etcétera. Por lo anterior, consideramos que la no inclusión de los lineamientos mínimos para la selección de los niños es un desacierto que debe subsanarse posteriormente.

Por otro lado, en relación con la participación de las niñas, niños y adolescentes en materia familiar, el Reglamento únicamente establece que deberá tomarse en cuenta (suponemos que según la edad y el grado de madurez) la opinión de los menores susceptibles de adopción, una vez que haya concluido la etapa de acogimiento preadoptivo, a fin de establecer si la adopción es o no conveniente (artículo 79). En este caso, pensamos que la participación del menor se ve reducida, y su opinión no es determinante.

En el aspecto de la participación de las niñas, niños y adolescentes, el Reglamento debió ser más amplio y señalar, o por lo menos delinear, mecanismos que permitan la participación en otros ámbitos de gran importancia, como el aspecto escolar.

Con respecto a la convivencia familiar, el Reglamento es acertado al establecer medidas especiales de acogimiento de menores, como son los centros de asistencia social y las familias de acogida. Asimismo, resulta un acierto del Reglamento establecer mecanismos de certificación y supervi- 
Esta revista forma parte del acervo de la Biblioteca Jurídica Virtual del Instituto de Investigaciones Jurídicas de la UNAM

sión de centros de asistencia y familias de acogida tratando de garantizar las mejores condiciones para los menores.

Conforme a lo anterior, la Procuraduría Federal deberá integrar el Registro Nacional de Centros de Asistencia Social, que deberá contener diversa información relativa a la identificación de los centros e información respecto al tipo de centro y sobre los resultados de las visitas de supervisión. El Registro permitirá conocer primero el número de centros de asistencia tanto públicos como privados, y así hacer una estimación de la población infantil que se encuentra en dichos centros.

De igual manera, consideramos que el Reglamento es acertado al momento de desarrollar el tema de la adopción, tanto nacional como internacional.

En primer término, pensamos que es un acierto del Reglamento señalar los requisitos con los que debe contar una familia para ser considerada como idónea para adoptar. El Reglamento especifica, en varios artículos, los requisitos sociales, económicos, psicológicos con que deben contar las familias que deseen adoptar, además de que el mismo Reglamento prevé un sistema de orientación y capacitación a través del cual se orienta a los futuros padres sobre las consecuencias sociales, económicas, psicológicas de la adopción.

El Reglamento también prevé como una etapa del proceso la convivencia preadoptiva; sin embargo, como ya lo mencionamos más arriba, el Reglamento no especifica la duración de la misma, y tan sólo señala una visita de un profesional para pronunciarse sobre la pertinencia de la adopción. La etapa preadoptiva es una fase de convivencia que pretende la adaptación familiar y afectiva del menor con los futuros padres, por lo que una única visita del profesional quizá no refleje el grado de adaptación.

En el mismo tenor de ideas, el Reglamento es omiso en señalar los procedimientos de seguimiento después de la adopción. El Reglamento no señala nada con respecto a los niños adoptados; únicamente señala que deberá haber un Registro de niños adoptados, que incluirá los datos generales del menor.

Propiamente en cuanto a la adopción internacional, como ya lo señalamos, el Reglamento recalca la posibilidad de que México puede fungir tanto como país de origen o emisor de menores como de recepción de éstos. En conexión con ello, el Reglamento adopta un punto de conexión más acorde con la legislación internacional, pues señala como punto de 
Esta revista forma parte del acervo de la Biblioteca Jurídica Virtual del Instituto de Investigaciones Jurídicas de la UNAM

conexión o de contacto la residencia habitual, tal como lo hace la Convención de La Haya de 1993, y no la nacionalidad, como lo hace la legislación local del Distrito Federal.

En cuanto al tema de los menores migrantes, el Reglamento tiene nuevamente un acierto cuando establece los datos que deben fijarse en la base de datos de niñas, niños y adolescentes, lo cual permitirá realizar estadísticas sobre la migración infantil y establecer políticas públicas para atender dicha situación.

En definitiva, el Reglamento de la LGDNNA es una gran contribución a la protección de los derechos humanos de las niñas, niños y adolescentes; no obstante, contiene algunos desaciertos, que es necesario resolver. Celebramos que por primera vez se vea a la niñez como sujetos de derechos y con capacidad de ejercerlos.

\section{GONGLUSIONES}

$\mathrm{Al}$ presente comentario legislativo le hemos dado un planteamiento un tanto familiar, aunque siempre desde una perspectiva de género, pero que adolece, por cuestiones de espacio, de una profundidad, aquella que amerita la visión de género, fundamental e imprescindible. La Ley y su Reglamento no tienen realmente una perspectiva de género, algo realmente lamentable.

El tema de la protección de los derechos de los menores ha ido tomando una importancia creciente en las últimas décadas en las sociedades modernas, y la nueva Ley General de los Derechos de Niñas, Niños y Adolescentes y su Reglamento es un reflejo de que México no piensa mantenerse al margen, máxime cuando el país está compuesto por una sociedad joven con un importante porcentaje de población infantil.

Entre los avances importantes están:

A. La creación del Sistema Nacional de Protección Integral de los Derechos de Niñas, Niños y Adolescentes como plataforma para la generación de políticas públicas nacionales, al que se le debe dar un presupuesto suficiente, y así, con la voluntad que ello supone, poder constituirse como un sistema eficiente - con recursos humanos cualificados - ; no obstante, en 2015 ya sucedió lo que se temía; es decir, no se etiquetó presupuesto suficiente para el Sistema, y ha quedado sin operar como debía. No obstante, para no anclarnos en el mal general de la falta de presupuestos con 
Esta revista forma parte del acervo de la Biblioteca Jurídica Virtual del Instituto de Investigaciones Jurídicas de la UNAM

perspectiva de protección a la minoridad, también debemos expresar que su eficiente desempeño dependerá de la coordinación de las autoridades de los tres niveles de gobierno en el cumplimiento del objeto de la Ley, así como de la asignación de recursos destinados a implementar las acciones legales correspondientes en todo el territorio nacional.

B. La obligación legal impuesta a las autoridades federales, de las entidades federativas y municipales, en el ámbito de sus respectivas competencias, de adoptar las medidas necesarias para garantizar los derechos de los menores sin discriminación de ningún tipo o condición. Es un tema recurrente en la ley el procurar un entorno que provea los apoyos necesarios para que niñas, niños y adolescentes con discapacidad para fomentar la inclusión y prevenir su discriminación.

C. El reconocimiento expreso de que los derechos de la niñez presentan una naturaleza interdependiente, indivisible y progresiva, lo que se traduce en que la vulneración de un derecho necesariamente impacta negativamente a los demás derechos; por ello, se debe promover una protección integral.

D. La regulación de la situación jurídica de niñas, niños y adolescentes migrantes, muy ad hoc con la triste realidad, ya que en los últimos años el tema migratorio ha representado una verdadera situación de crisis humanitaria, por los peligros a los que se enfrentan los menores cuando la extrema pobreza y/o violencia presente en sus lugares de origen los obliga a migrar a otros estados dentro de la República o tomar el rumbo hacia el norte para intentar ingresar ilegalmente a los Estados Unidos de América, peligroso viaje que muchas veces emprenden solos, sin acompañamiento de ninguno de sus padres o familiares cercanos.

Por ello, resulta fundamental que la Ley y su Reglamento establezcan que en todos los casos de procedimiento administrativo migratorio las autoridades deberán respetar las garantías de debido proceso, y desempeñarán sus funciones teniendo como consideración primordial el principio del interés superior de la niñez, privilegiando la reunificación familiar.

E. La creación de un Registro Nacional de Centros de Asistencia Social del Sistema Nacional DIF, que sea público y consultable. Ciertamente, un paso fundamental en pro de los derechos de los menores más desprotegidos, sin duda aquellos que han sido abandonados o separados del entorno familiar y se encuentran bajo custodia en alguno de estos centros. Directamente relacionado con lo anterior está la obligación de los centros 
Esta revista forma parte del acervo de la Biblioteca Jurídica Virtual del Instituto de Investigaciones Jurídicas de la UNAM

de atención, de mantener un expediente completo de cada menor bajo su custodia, para efectos de que su situación sea revisada y valorada de manera particular, con miras a facilitar su reincorporación familiar o social, de igual forma que se garantice la protección de sus datos personales.

Con la efectiva centralización de los datos relativos a la niñez se sientan las bases para materializar la responsabilidad del Estado como garante del pleno ejercicio de los derechos de menores y como agente promotor de su respeto y protección objetivo de la ley. Ello lo decimos con base en la falta de estadísticas relacionadas con los menores albergados. En lo que respecta a México, es necesario reconocer que aun cuando se han realizado esfuerzos, todavía no se cuenta con información a nivel nacional del total de población infantil que vive en centros asistenciales, principalmente en las instituciones privadas. Una muestra de ese esfuerzo son las cifras reportadas en 2012 como resultado de la implementación de proyectos elaborados por los sistemas DIF estatales, al amparo del Programa de Atención a Familias y Población Vulnerable, en su vertiente Fortalecimiento a las Procuradurías de la Defensa del Menor y la Familia, a cargo del Sistema Nacional DIF, que contabilizó 725 albergues, de los cuales $14.2 \%$ fueron públicos y $85.8 \%$ privados, en los que se atendía a una población total de 16,893 niñas, niños y adolescentes.*

Sin más, sólo deseamos la mejor de la suerte a la nueva normativa a favor del sector más vulnerable de nuestra sociedad: la infancia.

* Diagnóstico del Programa Nacional de Asistencia Social 2014-2018 (PONAS), publicado en el DOF el 30 de abril de 2014, disponible en http://dof.gob.mx/nota_detalle.php?'co $\operatorname{dig}_{0}=5343103$ Efech $^{2}=30 / 04 / 2014$, consultado el 5 de mayo 2015. 